apparatus much impaired, directed his treatment to inch to an inch; it had a nodulated appearance, and these points, and with so much success that $\mathrm{Mr}$. $\mathrm{T}$. when cut into was perfectly white, homogeneous in said for weeks he had not slept so well, or felt so structure, and without any bands or striated appearcomfortable, as the first two or three nights after Mr. ance whatever. The pylorus itself was only slightly Parry saw him. Still, however, he did not gain thickened, and this thickening appeared to depend strength, and it was at this time that I was called in. entirely upon an hypertrophied state of the mucous

The treatment which we agreed upon adopting was membrane. a continuation of the plan which had been commenced by Mr. Parry : small doses of blue pill, extract of colocynth, and hyoscyamus, to regulate the bowels; the nitro muriatic acid, with decoction of taraxacum as a general tonic and with the view of acting upon the liver, which was evidently at fault; and the use of the assafœtida lavement, for the purpose of relieving the tympanitic state of the bowels; at the same time his diet was carefully regulated. At my subsequent visit, the following day, on a careful examination of the abdomen, I stated my opinion that there was fluid in its cavity; but the flatulent distension was so great, that it was impossible to be quite certain as to its existence. There was no tenderness on pressure at the epigastrium, or any other part of the abdomen, and no irregularity of surface could be ascertained.

[In consequence of the temporary indisposition of Mr. Parry for two or three days, the subsequent history of the case fell wholly under my own observation.]

I left Mr. T. comfortable on Saturday evening, complaining only of the distension, and of very slight tenderness at the caput cœcum coli. On Sunday morning I was called up to sec him at four, a.m., and found him with an excessively rapid pulse, which I could not count; the greatest anxiety depicted upon his countenance, and complaining of the most agonising pain over the whole abdomen, which was so tender that he could not bear the slightest touch. All these symptoms had come on about two hours previously.

He had a mustard plaster applied to the abdomen, and I ordered a dose of morphia to be taken immediately, and repeated if necessary. This seemed in some degree to relieve the extreme pain, but he became insensible about eight o'clock, and died at halfpast nine, a.m.

Mr. Milnthorpe assisted me at the examination of the body twenty hours after dealh.

On opening the abdomen, we found more than three quarts of turbid serum, in which were floating a few small flakes of lymph. The bowels were immensely distended. On the anterior border of the lesser curvature of the stomach we found two irregular openings, of about three-quarters of an inch in diameter, the immediate margins of which were as thin as a piece of paper. These perforations were at the distance of about an inch and a half from the pylorus, and were situated in the centre of a mass of cartilaginous substance, which involved the whole circumference of the stomach, for a distance of from three to four inches from the pylorus. The mucous membrane lining this diseased portion did not present any unhealthy appearance, excepting that in one or two small patches, and around the edge of the perforations, it was slightly turgid, and of an ashy hue. But the muscular coat seemed to have disappeared entirely, and to be replaced by what I have above described as cartilage. The thickness of this substance varied in different parts from a quarter of an

In some parts the serous coat of the stomach was involved in the cartilaginous degeneration, while in others it was quite healthy.

A ttached to it in different places, by short pedicles, were a large number of small, fatty tumors, varying in size from a pea to that of a filbert, each being contained in a fibro-serous capsule.

The right lobe of the liver was slightly enlarged, and its whole tissue much congested. The gall bladder was perfectly empty of bile, and its cavity almost entirely filled by an immense gall stone.

The rest of the abdominal viscera were perfectly healthy. There was about an ounce of serum at the base of the brain, about six drachms in the ventricles, and between the arachnoid and pia mater there were a few slight traces of serous effusion.

High Harrogate, June 19.

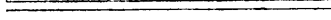 \\ CASE \\ OF}

DIVISION OF THE URETHRA. GONORRHOA-URETHRO-PLASTY.

\section{By M. Ricord.*}

The subject of this case, twenty-six years of age, was admitted into the Venereal Hospital of Paris, on the 16th of June, 1840. When only seven years of age, the patient had amused himself by tying a piece of thread round the penis, close to its root. The consequence was an urinary fistula, which persisted from the time of the accident up to the period of his admission into hospital. The parts, at this latter period, presented the following appearances:-The whole thickness of the spongy portion of the urethra is divided across, a little in front of the scrotum, thus separating the canal into two portions; the two orifices resulting from this division are separated from each other about 11.077 lines (French) by a deep, hard, cicatrix, which surrounds the whole penis like a ring; the urine and semen pass entirely through the artificial opening; erection is complete, but the sensations, during coition, are confined to the posterior portion of the canal.

On the lst of June, 1840, and eight days after connection, the patient was affected with gonorrhœa : the disease commenced in the posterior division of the urethra, and did not reach the anterior until four days afterwards.

June 17. The inflammation is excessively severe, and the discharge of a deep green colour, and very abundant; still the patient experiences pain only when the urine is passing over the posterior portion of the canal.

* A short account of this interesting case was published in the "Provincial Medical and Surgical Journal," Vol. II., 1840-41, p. 97. The complete history, for which we are indebted to the kindness of Mr. Acton, has just appeared in M. Ricord's lllustrations of the Venereal Disease. 
19. The acute attack has almost completely yielded to rest and an antiphlogistic regimen; there is still a little pain in making water. 'To have twenty-fuur scruples of cubebs in three doses; to continue his regimen, but omit the baths. On the 21 st the discharge from the posterior part of the urethra has considerably diminished, but that from the anterior remains unclianged.

24. The discharge from the posterior section has completely ccased. To continue the cubebs, and have better diet.

On the lst of July the use of the cubebs was suspended ; there is no discharge from the vesical portion of the urethra, while the state of the antcrior part does not seem to be changed.

July 6 . The discharge has reappeared in the vesical part of the canal; this may, perhaps, be explained by the fact that, during the use of the cubebs, the two artificial orifices were kept separated, but afterwards, this precaution being neglected, the matter from the anterior part came in contact with the posterior opening, and gave rise to a fresh infection.

8. The former treatment was adopted, and on the 16 th the discharge from the vesical portion of the urethra ceased. Treatment continued.

17. A solution of the nitrate of silver was injected into the anterior segment of the canal, and on the 20 th the discharge had nearly completely yiclded to the injections.

22. No trace of discharge; the treatment was suspended, and on the 26th the patient was allowed to leave the hospital for some time.

The patient having thus been cured of his gonorrhœa, it remained to make an attempt to relieve him from the effects of his accident; but before describing the operation which I performed for this purpose, I shall make a few clinical remarks on some important points connected with the case.

Although the majority of surgeons, at the present day, refuse to admit a specific seat of gonorrhœa, yet many think that the fossa navicularis is the point at which it always commences. An attentive examination of facts, however, proves that the disease commences at the point to which the infectious matter has been first applied.

The pain of gonorrhœa chiefly depends on the passage of urine over the inflamed parts, and on the erections which occur. Independently of these two causes, the mere inflammation of the mucous membrane of the urethra causes very little pain, as was seen in the present case.

The state of this patient also afforded an excellent opportunity for ascertaining the mode of action of certain remedies in gonorrhœa. It was shown in the clearest manner, that the remedies acted through the medium of the urine, after having passed through the circulation and the kidneys. Thus, on two different occasions, the curative action of the cubebs was exercised on that part of the urethra only over which the urine passed, while the anterior portion of the canal remained uninfluenced by it.

I do not mean by this to deny, as some have pretended that $I$ do, the general action of balsams; I admit that thcy may modify certain secretions, or communicate certain propertics to the blood, but experience has proved to me that in this respect their action is more uncertain and less efficient, as was shown by the continuation of the disease in the anterior part of our patient's urethra, and as is seen every day in the treatment of balanitis, purulent discharges from the vagina, gonorrhœal ophthalmia, \&c., where the balsams produce little or no effect. The action of these remedies in the latter cases is not specific, but merely revulsive, and when they are susperided the disease generally recurs.

The patient returned into hospital on the 20th of October, 1840, and on the 3rd of November I performed the following operation:-

Having placed him in the usual position for lithotomy, and introduced a sound with a wide groove through the orifice of the posterior portion of the urethra, I made an incision in the central linc of the perineum about $8 \frac{3}{4}$ lines in length, and commencing immediately behind the bulb of the urethra; the incision was carried into the urethra, and the latter divided to the extent of four lines; I now attempted, but in vain, to pass an instrument through the artificial opening, and at length succeeded with a female catheter, which I immediately fixed in its place.

My next step was to operate for the phymosis; I then refreshed the edges of the fistulous opening, and, when the bleeding ccased, I set about bringing the edges of the wound together. A sinall bougie was passed, through the meatus urinarius, down to the catheter, and the edges of the wound having been approximated in a transverse direction, were united with two alternate points of the twisted and common suture.

The patient was now placed in bed, the thighs being flexed and elevated on pillows; cold water dressing was applied to the wound, and two pills, containing camphor and opium, were administered; towards evening some reaction came on, and the patient was bled; the urine came away through the catheter in the perineum; on the following morning the urine still appeared to pass through the same channel, but the wound seemed a little swollen, and the ligatures moistened by the urine.

On the third day the sutures came away, and the urine flowed through the fistula as copiously as through the perineal opening; its edges, however, retained the transverse direction. On the sixth day the greater part of the urine passed through the fistulous orifices; the female catheter was therefore removed, and replaced by a larger gum-elastic catheter; on examining the female catheter, it was found that the orifice was completely obstructed by a clot of blood.

On the 17th an abscess formed in the scrotum, and was opened; the utmost care was now employed to prevent the urine from passing beyond the artificial opening in the perineum, but without success. This, however, was absolutely necessary, and in order to effect it, a longer catheter was passed into the perineal orifice; a bougie was also passed through the meatus urinarius, and brought out through the perineal orifice, but this likewise failed, and caused so much pain, that the patient removed it.

From this period, up to the 19th of January, the artificial opening in the perineum was gradually dilated by the introduction of larger and larger catheters; the abscess in the scrotum had now completely healed, and the urine escaped through the fistula in 
small quantities only, and at distant intervals. As / while the lower remained free, and a new canal was the patient became impatient, I was unable to wait formed, being merely somewhat shorter than the any longer, and therefore attempted to bring the edges original vagina. The woman recovered perfectly, of the fistula together a second time. The edges were and the functions of the uterus were soon restored.pared, and brought together by twisted sutures alone, Orvosi Tir, or the Hungarian Magazine of Health.

which passed through the whole thickness of the skin; a bougic, as in the former attempt, had been passed down to the bulb of the urethra. 'The penis was now covered with lint dipped in cold water, and injections of cold water were frequently thrown into the bladder. The urine came away frecly through the artificial upening in the perineum. On the 22 nd, threc days after the operation, the two external pins were removed; the edges of the wound appeared to be united; there was no trace of pus or of urine about it.

On the 24 th the two remaining pins wcre removed, but a small quantity of pus and urine now escaped through a small opening at one angle of the wound. On the lst of February this small orifice, which had been touched two or three times with lunar caustic, had completely healed up, but another one appeared at the opposite angle of the wound, and this continued for a long time in the shape of a capillary opening, scarcely visible to the naked cye. On the 12 th of February, three months and nine days after the first operation, the perineal catheter was removed, and a small catheter introduced into the bladder along the urethra. This was done with some difficulty, but immediately afterwards the wound in the perineum began to heal, and was completely cicatrised on the 2nd of March. On the 17 th of $\Lambda$ pril there was no longer any occasion to employ a catheter. During the whole of this period, the capillary orifice already mentioned was alternately touched with lunar caustic and tincture of cantharides.

When the patient left the hospital he was completely cured; the form and functions of the penis were restored to their normal conditions; but a month elapsed before the semen could pass completely through the meatus urinarius.

\section{COMPLETE PROLAPSUS AND SEPARATION OF THE VAGINA.}

A woman, 25 years of age, who laboured under some slight disorder occasioned by errors of diet, took an emetic on the fourth day, which produced copious vomiting, and relieved her greatly.

A few days afterwards she began to complain of burning pain during micturition, and had some dis. charge of blood from the vagina, with severe pain in the external organs of generation. On examination, the external parts were found in a state of gangrene; on the following day the patient complained of an unusual feeling about the parts, and it was found that the whole vagina was prolipsed; as it was impossible to return it, antiseptic remedies were merely applied. The exposed parts now sloughed, and were finally completely removed on division of a band which retained them superiorly. The fever and other unfavourable symptoms quickly subsided; to prevent adhesion of the parts, a piece of sponge, moistened with some aromatic decoction, was introduced into the vagina. The vaginal portion of the uterus now became adherent to the upper edge of the vagina, up in every corner of the empire-lull-grown like

\section{PROVINCIAI, MEDICAL JOURNAL}

\section{SATURDAY, JULY 2, 1842.}

Experience has abundantly proved that partial reform of long-standing abuses is to be deprecated almost as much as the absence of any reform at all. Piece-meal legislation and vaccilating policy are not only evidences of a weak and incapable government, but often inflict greater damage on the commonwealth than actual abuse of power or complete neglect.

It is, therefore, sincerely to be hoped that any attempt to reorganise the medical profession in this country will be based on general views-on careful consideration of what is required by the public and the profession at large-not on the narrow and selfish propositions which may emanate from any particular corporation, or obtain the support of any one class of medical practitioners.

The question of medical reform, which appeared to slumber since the unsatisfactory termination of the medical conference of last year, has been revived, in opportune season, by the pamphlet of Sir James Clark. The question has not been abandoned, nor has the zeal of its advocates undergone a change. Time, instead of allaying discontent, only increases our dissatisfaction, and every day adds fresh proof of the necessity - the absolute and pressing necessity - of a complete revision of the laws which are supposed to regulate the medical profession throughout the United Kingdom.

That such necessity exists, that an earnest and urgent desire of change is felt by the great majority of the profession, cannot be denied by any one who has paid the slightest attention to the subject. Formerly we thought that the licensing bodies were confined in number to nineteen, the Archbishop of Canterbury being included in the goodly array. The poor.law commissioners, however, have discovered that " the ordinaries of the several dioceses in England and Wales possess the power of granting licenses to practise medicine within their respective dioceses." Thus have we a legion of licensers added to the respectable number of nineteen, and the question of medical qualification involved in the most curious and inextricable complication. No wonder that the poor-law commissioners, aided by the law officers of the crown, should find it difficult to distinguish the sheep from the goats, or that docturs should spring 